INPLASY

PROTOCOL

To cite: Yang et al. The protocol for meta-analysis of Tai Chi in the prevention and treatment of osteoporosis. Inplasy protocol 202190102. doi:

10.37766/inplasy2021.9.0102

Received: 27 September 2021

Published: 27 September 2021

Corresponding author:

Mao Yang

drmaoyang@163.com

Author Affiliation:

The First Affiliated Hospital of Anhui University of Chinese Medicine.

Support: None.

Review Stage at time of this submission: The review has not yet started.

Conflicts of interest:

None declared.

\section{The protocol for meta-analysis of Tai Chi in the prevention and treatment of osteoporosis}

\author{
Yang, $\mathrm{M}^{1}$; Zhao, J2; Fang, Z33.
}

Review question / Objective: Can Tai Chi prevent and treat osteoporosis?

Condition being studied: Osteoporosis.

Eligibility criteria: (1) Randomized controlled trials; (2) The subjects were patients with osteoporosis and osteopenia or people who were involved in the prevention of osteoporosis and osteopenia; (3) In the study, the treatment group must include Tai Chi, the control group is not limited, but must not include exercise. Both groups can be allowed to include the same exercise interventions; (4) The primary outcomes are the bone mineral density of lumbar spine and femoral neck. The secondary outcomes included bone mineral density (except other parts of lumbar spine and femoral neck), effective rate, bone metabolic markers and adverse events. When multiple time points are reported in a study or in multiple articles of the same study, the longest follow-up period for treatment was considered in our article. And if overlapping groups of participants are reported in different studies, literature with high quality or large sample size is selected as the subjects. Full texts of all references were available.

INPLASY registration number: This protocol was registered with the International Platform of Registered Systematic Review and Meta-Analysis Protocols (INPLASY) on 27 September 2021 and was last updated on 27 September 2021 (registration number INPLASY202190102).

\section{INTRODUCTION}

Review question / Objective: Can Tai Chi prevent and treat osteoporosis?

Condition being studied: Osteoporosis.

\section{METHODS}

Participant or population: Osteoporosis patients.

Intervention: Tai Chi. 
Comparator: Other interventions excluding exercise.

Study designs to be included: Randomized controlled trial.

\section{Eligibility criteria: (1) Randomized} controlled trials; (2) The subjects were patients with osteoporosis and osteopenia or people who were involved in the prevention of osteoporosis and osteopenia; (3) In the study, the treatment group must include Tai Chi, the control group is not limited, but must not include exercise. Both groups can be allowed to include the same exercise interventions; (4) The primary outcomes are the bone mineral density of lumbar spine and femoral neck. The secondary outcomes included bone mineral density (except other parts of lumbar spine and femoral neck), effective rate, bone metabolic markers and adverse events. When multiple time points are reported in a study or in multiple articles of the same study, the longest follow-up period for treatment was considered in our article. And if overlapping groups of participants are reported in different studies, literature with high quality or large sample size is selected as the subjects. Full texts of all references were available.

Information sources: CNKI, Pubmed, Web of Science, EMbase, Cochane.

Main outcome(s): The bone mineral density of lumbar spine and femoral neck.

Quality assessment / Risk of bias analysis: Assessing the risk of bias of RCTs in this review used the Cochrane Collaboration Risk of Bias Tool. And risk of bias was assessed according to the Cochrane Handbook. For each included study, each type of bias was categorized as high, low, or unclear and entered into the risk of bias table. Two reviewers independently assessed the risk of bias of the included studies. Any discrepancies were resolved by consensus, including input from a third party if required.
Strategy of data synthesis: The outcomes of interest include dichotomous data and continuous variables, dichotomous data were presented as the relative risk and mean difference (MD) was used to express the continuous variables. Meanwhile, standardized mean difference (SMD) was chosen if clinical outcome was the same but measured using different methods, evaluation criterion or the baselines of the studies are inconsistent in the different trials. The $95 \%$ confidence interval $(\mathrm{Cl})$ was computed. Heterogeneity across studies was quantified by the 12 statistic. An $12>$ $50 \%$ was considered to represent the possibility of statistical heterogeneity, and the random-effects model was used in this meta-analysis. Otherwise, no obvious heterogeneity $(12<50 \%)$ was considered to have occurred in the included studies, and the fixed-effects model was used. The forest plot for each parameter was constructed to illustrate the weight ratio of each incorporated study. Each article one by one was excluded to evaluate the sensitivity of the meta-analysis, and to compare the differences in the combined effects before and after. If the pooled results are reversed after exclusion, the results of this meta-analysis may be unstable. All statistical analyses were carried out using the RevMan5.3 software, and the significance threshold was a 2sided $P<.05$. According to Cochrane Handbook 5.3, if Inclusion studies include a study with multiple intervention groups, the recommended method in most situations is to combine all relevant experimental intervention groups of the study into a single group and to combine all relevant control intervention groups into a single control group.

\section{Subgroup analysis: Not used.}

Sensitivity analysis: Each article one by one was excluded to evaluate the sensitivity of the meta-analysis, and to compare the differences in the combined effects before and after. If the pooled results are reversed after exclusion, the results of this metaanalysis may be unstable.

Country(ies) involved: China. 
Keywords: TaiChi osteoporosis, metaanalysis.

Contributions of each author:

Author 1 - Mao Yang.

Author 2 - Jindong Zhao.

Author 3 - Zhaohui Fang. 